\title{
DNAJB6 Suppresses Alpha-synuclein Induced Pathology in an Animal Model of Parkinson's Disease
}

\section{Sertan Arkan}

Lund University https://orcid.org/0000-0003-2581-5620

\section{Mårten Ljungberg}

Lund University: Lunds Universitet

\section{Deniz Kirik}

Lund University: Lunds Universitet

Christian Hansen ( $\nabla$ christian.hansen@med.lu.se )

Lund University https://orcid.org/0000-0002-2891-6876

\section{Research article}

Keywords: Protein homeostasis, Parkinson`s Disease, Alpha-synuclein, Adeno Associated Virus, DNAJB6, Heat Shock Protein 70

Posted Date: November 24th, 2020

DOl: https://doi.org/10.21203/rs.3.rs-112939/v1

License: (9) This work is licensed under a Creative Commons Attribution 4.0 International License. Read Full License

Version of Record: A version of this preprint was published at Neurobiology of Disease on October 1st, 2021. See the published version at https://doi.org/10.1016/j.nbd.2021.105477. 
DNAJB6 suppresses alpha-synuclein induced pathology in an animal model of Parkinson's Disease

Sertan Arkan ${ }^{1}$, Mårten Ljungberg ${ }^{1}$, Deniz Kirik ${ }^{2}$ and Christian Hansen ${ }^{1}$

${ }^{1}$ Lund University, Molecular Neurobiology, Department of Experimental Medical Science, BMC B11, 221 84, Lund, Sweden.

${ }^{2}$ Brain Repair and Imaging in Neural Systems, Department of Experimental Medical Science, Lund University, BMC D11, Lund 22184, Sweden

Sertan Arkan: sertan.arkan@med.lu.se

Mårten Ljungberg: mårtenljungberg@gmail.com

Deniz Kırık: deniz.kirik@med.lu.se

Correspondence should be addressed to Christian Hansen (christian.hansen@med.lu.se) 


\begin{abstract}
Background: $\alpha$-synuclein ( $\alpha$-syn) aggregation can lead to degeneration of dopaminergic neurons in the substantia nigra pars compacta $(S N p c)$ as invariably observed in patients with Parkinson's Disease (PD). The co-chaperone DNAJB6 has previously been found to be expressed at higher levels in PD patients than in control subjects and was also found in Lewy bodies. Our previous experiments showed that knock out of DNAJB6 induced $\alpha$-syn aggregation in cellular level. However, effects of overexpression of DNAJB6 against $\alpha$-syn aggregation remains to be investigated.
\end{abstract}

Methods: we used to $\alpha$-syn CFP/YFP HEK293 FRET cell line to investigate the effects of overexpression of DNAJB6 in cellular level. $\alpha$-syn aggregation was induced by transfection $\alpha$-syn preformed fibrils (PPF), then was measured FRET analysis. We proceeded to investigate if DNAJB6b can impair $\alpha$-syn aggregation and toxicity in an animal model and used adeno associated vira (AAV6) designed to overexpress of human wt $\alpha$-syn, GFPDNAJB6 or GFP in rats. These vectors were injected into the SNpc of the rats, unilaterally. Rats injected with vira to express $\alpha$-syn along with GFP in the SNpc where compared to rats expressing $\alpha$-syn and GFP-DNAJB6. We evaluated motor functions, dopaminergic cell death, phosphorylated $\alpha$-syn in SNpc and axonal degeneration in striatum.

Results : We show that DNAJB6 prevent $\alpha$-syn aggregation induced by $\alpha$-syn PFF's, in a cell culture model. Also, we observed $\alpha$-syn overexpression caused dopaminergic cell death and that this was strongly reduced by co-expression of DNAJB6b. In addition, the lesion caused by $\alpha$-syn overexpression resulted in behavior deficits, which increased over time as 
seen in stepping test, which was rescued by co-expression of DNAJB6b. Moreover, we observed an increase in serine 129 (S129) phosphorylated $\alpha$-syn in $S N p c$ of rats overexpressing $\alpha$-syn, that was lowered by DNAJB6 co-expression.

Conclusion : We here demonstrate for the first time that DNAJB6 is a strong suppressor of $\alpha$ syn aggregation in cells and in animals and that this results in a suppression of dopaminergic cell death and PD related motor deficits in an animal model of PD.

Keywords: Protein homeostasis, Parkinson`s Disease, Alpha-synuclein, Adeno Associated Virus, DNAJB6, Heat Shock Protein 70 


\section{Backgroud}

Parkinson's disease (PD) is the most common neurodegenerative movement disorder. It is primarily caused by a selective loss of dopaminergic neurons in the substantia nigra pars compacta $(S N p c)(1-4)$. This neurodegenerative process correlates with the formation of large protein-rich cytoplasmic inclusions, known as Lewy bodies (LBs), in which aggregated $\alpha$-syn is main protein component (2-5). The $S N C A$ gene, which encodes $\alpha$-syn, is also linked to familial forms of $\mathrm{PD}$, caused by missense mutations, which result in increased aggregation of the protein. It is therefore believed that $\alpha$-syn aggregation plays a key role in PD pathogenesis. $\alpha$-syn is abundantly expressed in neurons and plays a role in exocytosis(6-8). $\alpha-$ syn does not normally form large aggregates in neurons, despite that it is highly expressed, because of an effective protein homeostasis system(9-12). $70 \mathrm{kDa}$ heat shock protein (Hsp70) plays a major protective role against protein aggregation and can prevent aggregation of $\alpha$-syn (9, 13-15). However, a large body of work has shown that Hsp70 does not directly recruit protein substrates in vivo, but do so with help from co-chaperones, such as the DNAJ proteins $(16,17)$. DNAJB6, is expressed in neurons, and has been found to be present in LBs of PD patients (18). We have previously identified that DNAJB6 is a suppressor of $\alpha$-syn aggregation in a cell line model and in in vitro based studies, in a HSP70 dependent manner (19). In support of this, we also found that DNAJB6 prevents $\alpha$-syn aggregation induces by $\alpha$ syn preformed fibrils (PFFs), using an unbiased $\alpha$-syn FRET system to quantify $\alpha$-syn aggregation (20). Other labs have shown that, DNAJB6b suppress aggregation of multiple amyloid proteins in cells (21-23) and recently it shown that DNAJB6b suppresses disease progression, in an HD mouse model (24). As we have so far only explored if DNAJB6 is a 
suppressor in non-neuronal cell line models and in vitro, we wanted to explore if DNAJB6 could prevent $\alpha$-syn induced behavioral deficits and toxicity in an $\alpha$-syn overexpression based animal model of PD. Here we show that DNAJB6b does indeed impair $\alpha$-syn induced behavioral deficits by preventing $\alpha$-syn induced death of dopaminergic neurons in the $S N p c$.

\section{Materials and Methods}

\section{Cell Culture, plasmids and transfections.}

HEK293 cells stably expressing $\alpha$-syn-CFP $\alpha$-syn-YFP were a kind gift from professor Marc Diamond and were generated as described in (25). Cells were maintained in Dulbecco's Modified Eagle's Medium with GlutaMAX-I (DMEM) (Thermo Fisher Scientific, US), 10\% heat inactivated fetal bovine serum (FBS) (Thermo Fisher Scientific, US) and $1 \%$ penicillin/streptomycin (P/S) (Thermo Fisher Scientific, US) and incubated at $37{ }^{\circ} \mathrm{C}, 5 \% \mathrm{CO}_{2}$ in humidified condition. Lipofectamine Ltx was used for transfection of mCherryDNAJB6 and mCherry plasmids according to manufacturer protocol (Thermo Fisher Scientific, US) 72 hours prior to FRET analysis and Lipofectamine 2000 was used to transfect pre-formed $\alpha$ synuclein fibrils at $10 \mathrm{nM}$ final concentration 48 hours prior to FRET analysis. Preformed fibrils synthesis and transfection were performed as described in (20). mCherry expression plasmid was generated by replacing mCherry with AcGFP in the pAcGFP-C1 vector using restriction enzymes and the mCherry-DNAJB6 was subsequently generated by amplifying human DNAJB6b cDNA using primers 5'gtcactcgagctatggtggattactatgaagttctaggcgtg3' and 5' gtcaggatccttacttgttatccaagcgcagcagctg3' which contained restriction enzyme overhangs, followed by insertion of the cDNA into the pmCherry-C1 vector using the restriction enzymes XhoI and BamHI.

\section{Florescence Resonance Energy Transfer (FRET)}

FRET experiments were performed on a BD LSR Fortessa with the software BD FACS Diva 8.0.1 (BD Biosciences, US). Gates for analysis were calibrated using single stained controls as 
well as negative controls. YFP was excited using a $488 \mathrm{~nm}$ laser and emitted light was detected using a 530/30 bandpass filter. CFP was excited at $405 \mathrm{~nm}$ and the emitted light was detected using a 442/46 bandpass filter. mCherry was excited at $488 \mathrm{~nm}$ and the emitted light was detected using 610/20nm bandpass filter. FRET was measured by excitation of CFP using a 405 laser and the fluorescence was captured using 525/50 bandpass filter.

\section{Western Blot}

The cells were washed with phosphate buffered saline (PBS) and subsequently lysed in a lysis buffer $(0.5 \%$ Triton $\mathrm{X}-100, \quad 50 \mathrm{mM} \quad$ Tris $\mathrm{HCl}, \quad 175 \mathrm{mM} \quad \mathrm{NaCl}$ and $5 \mathrm{mM}$ Ethylenediaminetetraacetic acid (EDTA), pH 8, 1:100 protease inhibitor cocktail (SigmaAldrich, US) on ice for at least 15 minute (min). The cell debris was spun down at 15,000 rcf for $10 \mathrm{~min}$ at $4 \mathrm{C}$, and the supernatant was collected. Cell lysates mixed with $25 \% 4 \mathrm{x}$ Laemmli buffer containing $10 \%$ of $0.1 \mathrm{M}$ Dithiothreitol (DTT), and boiled for $5 \mathrm{~min}$ at $96{ }^{\circ} \mathrm{C}$. $25 \mathrm{ug}$ of proteins from lysate samples were separated on 10\% SDS-PAGE polyacrylamide gels, then the samples transferred onto Polyvinylidene fluorid (PVDF) membranes using the Trans-Blot Turbo Transfer System (Bio-Rad, US). The membrane was blocked with 5\% skimmed milk powder (Sigma Aldrich, US) dissolved in PBS containing 0.05\% Tween (PBS-

T) for 1 hour (h) at room temperature (RT), after which they were washed thrice for 5 min with PBS-T at RT. Subsequently, the membrane incubated in $2 \%$ skimmed milk in PBS-T with 1:2000 anti-DNAJB6 primary antibody (Proteintech, US) overnight shaking in $4{ }^{\circ} \mathrm{C}$. The next day, the membrane was washed thrice in PBS-T for $5 \mathrm{~min}$ and then incubated shaking at RT for $1 \mathrm{~h}$ in 3\% skimmed milk in PBS-T with 1:5000 anti-rabbit secondary antibody (Dako, Denmark). The membrane was washed then thrice for $5 \mathrm{~min}$ in PBS-T. Western Blotting Luminol Reagent (Santa Cruz Biotechnology,US) was used for chemiluminescence reactions and protein bands were developed using a ChemiDoc ${ }^{\mathrm{TM}}$ XRS + Molecular Imager (Bio-Rad, US) and the Image Lab software (Bio-Rad,US). After stripping the membrane for 15 min at 
RT (Restore Buffer, Thermo Fisher Scientific, US). The membrane was washed twice with PBS-T for 5 min and then blocked in 5\% skimmed milk in PBS-T for 30 min before being incubated with a 1:10000 HRP coupled anti- $\beta$-actin primary antibody (Sigma Aldrich, US) and developed as described above.

\section{Animal Housing}

Female Sprague-Dawley rats (imported at a weight of 225 to 250 g; Charles River Germany) were housed with ad libitum access to food and water under a 12-h light/dark cycle.

\section{Experimental Design}

The rats were unilaterally injected with either cocktails of AAV6- human wild type (wt) $\alpha$-syn and AAV6-GFP or AAV6- human wt type $\alpha$-syn and AAV6 DNAJB6-GFP vectors into the SNpc. Cylinder and stepping tests performed just before stereotaxic injection (to create a baseline control) and after 4 weeks, 8 weeks and 12 weeks, to evaluate the motor function of the rats. After completing the behavioral tests, the rats were euthanized and immunohistochemical (IHC) staining were performed.

\section{rAAV vector production}

An AAV6 vector expressing human wild-type $\alpha$-syn under the human synapsin- 1 promoter was used in this study as described $(26,27)$. The vector titer of $\alpha$-syn and GFP mixture was $4.4 \times 10^{13}$ human wt $\alpha$-syn and GFP-DNAJB6 vector mixture was $4.4 \times 10^{13}$ genome copies/mL. AAV6 GFP- DNAJB6 vector was produced in Vigene Bioscience (US).

\section{Stereotaxic Injections}

The rats were anesthetized by intraperitoneal injection of $6 \mathrm{~mL} / \mathrm{kg}$ of a $20: 1$ mixture of Fentanyl and Dormitor (Apoteksbolaget, Sweden). After placing the animal into a stereotaxic frame (Stoelting, Wood Dale, USA), 2 ul of rAAV6 vector cocktails were unilaterally injected into the substantia nigra (SN) using the following coordinates from: anteroposterior (AP) -5.0 $\mathrm{mm}$, mediolateral (ML) -2.0 mm from bregma and dorsoventral (DV) -7.2 mm from the dura. 
The tooth bar was adjusted to $-2.3 \mathrm{~mm}$. Injection was performed using a pulled glass capillary (Stoelting, Wood Dale, USA) fixed into a $5 \mathrm{uL}$ Hamilton syringe with a blunt 22s gauge needle. The viral vectors injected at a constant rate $(0.1 \mathrm{uL}$ every $15 \mathrm{~s})$, the glass capillary was held in place for $5 \mathrm{~min}$, subsequently, retracted $0.1 \mathrm{~mm}$ and after 1 min it was slowly withdrawn from the brain. Temgesic and anti-sedan (Apoteksbolaget, Sweden) were administered subcutaneously as analgesic and to reverse anesthesia.

\section{Behavioral Tests}

Behavioral tests were performed before stereotaxic injections as control, and after at 4 weeks, 8 weeks, and 12 weeks by the same researcher.

Stepping Test: Stepping test was performed to evaluate forelimb akinesia as described in (28). Briefly, the number of steps counted by the left and right forepaws when passively moved along a $90 \mathrm{~cm}$ trajectory in forehand and backhand directions. The rats were trained to complete the test prior to experiments and the average of 3 trials were used in statistical analysis. The data are presented contralateral forehand forepaw contacts as percentage of right forehand forepaw contacts

Cylinder Test: The cylinder test was used to evaluate the motor functions of rats as described in (28). The rats were allowed to move freely in a clear glass cylinder. Mirrors were placed behind the cylinder to be able to observe all forelimb contacts on the glass wall. The videotapes were evaluated by an observer to the identity of the animals and the number of left and right paw touches on the cylinder wall were counted for 25 contacts. The data are presented contralateral (left) forepaw contacts as percentage of total paw contacts

\section{Histological analysis}

The rats were euthanized at 12 weeks after AAV injection by an overdose of sodium pentobarbital and perfused via the ascending aorta first with $50 \mathrm{~mL} / \mathrm{min}$ of $0.9 \% \mathrm{NaCl}$ for 1 min followed by $50 \mathrm{~mL} / \mathrm{min}$ of ice-cold $4 \%$ paraformaldehyde (PFA in $0.1 \mathrm{M}$ phosphate 
buffer, pH7.4) for 5 min. Brains were removed and post-fixed in 4\% PFA overnight and then transferred into $25 \%$ sucrose for cryoprotection at $4{ }^{\circ} \mathrm{C}$ for a minimum of 2 days. Subsequently, the brains were cut into 40 um thick 8 series of coronal sections on a microtome (Leica, Germany) and stored in an anti-freeze solution ( $0.5 \mathrm{M}$ phosphate buffer, $30 \%$ glycerol, $30 \%$ ethylene glycol) at $-20{ }^{\circ} \mathrm{C}$. IHC stainings of tyrosine hydroxylase $(\mathrm{TH})$ were performed as free floating sections at RT. The sections were washed from anti-freeze solution using tris-buffered saline (TBS) buffer ( $5 \mathrm{mM}$ Tris, $15 \mathrm{mM} \mathrm{NaCl}$ ). The endogenous peroxidase activity in the sections were quenched by incubation in $3 \% \mathrm{H}_{2} \mathrm{O}_{2}$ (Sigma Aldrich) and 10\% methanol in TBS buffer for $30 \mathrm{~min}$. After thrice washes with TBS buffer, sections were incubated in $0.05 \%$ Triton X-100 (Sigma Aldrich) in TBS buffer (TBS-T) containing $5 \%$ of rabbit normal serum (Vector Laboratories Inc, US) matching the species used to raise the secondary antibody for that protocol. This was performed for $1 \mathrm{~h}$ to eliminate non-specific secondary antibody binding. Anti-sheep TH primary antibody (Abcam, US) was diluted as 1:2000 in $1 \%$ BSA in TBS-T overnight at RT. The second day, the sections were thrice washed with TBS-T for $10 \mathrm{~min}$ and incubated with the biotinylated anti-sheep secondary antibody (1:200, Vector Laboratories Inc, US) in 1\% BSA in TBS-T for $1 \mathrm{~h}$. Sections were again washed with TBS-T for 10 min and incubated with an avidin-biotin-peroxidase complex solution (Vectastain ABC kit, Vector Laboratories Inc, US) for 1 h. The specific staining was visualized using 3,30-diaminobenzidine (DAB Safe, Saveen Werner, Sweden) and 0.01\% $\mathrm{H}_{2} \mathrm{O}_{2}$. For preservation and visualization sections were mounted on chromatin-gelatin coated glass slides, dehydrated in increasing alcohol solutions, cleared in xylene and coverslipped using DPX (Sigma-Aldrich, Sweden).

\section{Immunofluorescence stainings}


Immunofluorescence stainings were performed with sheep anti-TH (Abcam, cat\# ab118 (working dilution 1:2000)), mouse anti $\alpha$-syn (BD bioscience, cat\# 610787 (working dilution 1:1000)) and rabbit anti-p129 $\alpha$-syn (Abcam, Cat\#51253 (working dilution 1:1000)) antibodies. The free-floating sections were washed thrice with PBS from anti-freeze solution. The sections were then incubated in $0.025 \%$ Triton X-100 (Sigma Aldrich) in PBS containing $5 \%$ of normal serums (Vector Laboratories Inc, US) matching the species used to raise the secondary antibody for that proper stainings for $1 \mathrm{~h}$. Primary antibodies were diluted in PBS and overnight incubated at $4{ }^{\circ} \mathrm{C}$. The next day, the sections were washed thrice with PBS for 5 min, then incubated with Cy3 and Cy5 (working dilution 1:300) fluorophore conjugated secondary antibodies (Jackson Immunoresearch, Sweden). The sections were mounted on positive charged superfrost plus glass slides (ThermoScientific, US) and cover-slipped using PVA/DABCO. The immunofluorescence stainings were visualized on a Leica SP8 laserscanning confocal microscope (Leica, Germany).

\section{Cell counting analysis}

Tyrosine hydroxylase positive $(\mathrm{TH}+)$ neurons were manually quantified in only a specific section that $\mathrm{SN}$ is separated from ventral tegmental area by medial terminal nucleus of accessory optic system (AP: -5.20 from Bregma). Light microscope (Zeiss Primostar, Germany) was used to visualize neurons. Data presented means \pm SEM and expressed as percent value of the contralateral side of the section.

\section{Optical Densitometry}

Optical density of $\mathrm{TH}+$ fibers was analysed on digital images of coronal striatal sections obtained using a microscope (Nikon Eclipse 80 İ, Japan). For each animal the optical density was measured at seven rosto-caudal levels over the whole striatum according to the atlas of Paxinos and Watson (1998): (1) AP, +1.6; (2) AP, +1.0; (3) AP, +0.2; (4) AP, -0.3 ; (5) AP, -0.9 ; (6) AP, -1.4 ; and (7) AP, -2.1 relative to bregma. - was outlined using IMAGEJ 
software (version 1.50i, NIH, USA). Optical density readings were corrected for non-specific background using density measurements from the corpus callosum of each animal. Data presented are means \pm SEM from all brains and expressed as percent of contralateral side values.

\section{Statistical Analysis}

All behavioral data were analyzed by repeated measure two-way ANOVA followed by post hoc Bonferroni test. Counting of TH positive neurons in the $S N p c$, quantification of the optical fiber density in the striatum and quantitative FRET measurements of cell culture experiments were analyzed by independent $t$ test (Graphpad Prism 6, US), assuming normal distribution. $P<0.05$ were considered statistically significant. All data are expressed as mean \pm SEM.

\section{Results}

\section{Overexpression of DNAJB6 is protect against $\alpha$-syn aggregation in cellular model of PD}

We have previously shown that $\alpha$-syn PFF seeded $\alpha$-syn aggregation was increased in a HEK293 cell line, stably expressing $\alpha$-syn-CFP and $\alpha$-syn-YFP (hereafter named $\alpha$-syn FRET cells), when DNAJB6 was knocked out (20). Here, we examined if overexpression of DNAJB6 would suppress $\alpha$-syn aggregation induced by PFF's in the same cell line set up, and observed that overexpression of DNAJB6 decreased the seeded $\alpha$-syn aggregation more than 4-fold (Figure 1A and B). This observation showed that increasing DNAJB6 expression in cells protects against $\alpha$-syn aggregation.

\section{AAV6 mediated overexpression of DNAJB6 rescue to cell death in SN}

Next, we wanted to investigate if the protective effect of DNAJB6 against $\alpha$-syn aggregation seen in cells, could also protect against toxic $\alpha$-syn aggregation in an $\alpha$-syn overexpression based animal model of PD. We therefor set up a rat model, in which we induced $\alpha$-syn overexpression in the $S N p c$ by use of viral vectors incorporated into AAV6 vira. Two groups 
of 9 rats each were unilaterally injected with either AAV6/ human wt $\alpha$-syn and AAV/GFP (control) or AAV6/ human wt $\alpha$-syn and AAV/GFP-DNAJB6 vectors. Of these rats were 8 of 9 from each group showed successful targeting the $S N p c$, as shown in representative images (Figure 2A-H). Rats co-injected with vector expressing $\alpha$-syn and GFP displayed far less TH positive neurons in injected $S N p c$ compared to non-injected control side $(\% 33.62 \pm 7.404, \mathrm{n}$ :8), whereas rats injected with vectors to induce expression of human wt $\alpha$-syn and GFPDNAJB6, showed substantially more TH positive cells in the injected side $(\% 61.34 \pm 8.162, \mathrm{n}$ :8), suggesting that DNAJB6 protects against $\alpha$-syn induced neuronal cell death (df $: 14, \mathrm{t}$ :2.515, $\mathrm{p}: 0.0247$ ) (Figure $2 \mathrm{~K}$ and L). While there was a clear difference in cell loss of the dopaminergic neurons in the $S N p c$ between the groups, TH staining of the striata revealed that there was some loss of $\mathrm{TH}+$ optical fiber density in both groups of rats on injected side compared to contralatereal control striata, but also that the TH density loss was similar (df $: 14, \mathrm{t}: 0.06363, \mathrm{p}: 9502)$ in both human wt $\alpha$-syn and GFP $(\% 62.74 \pm 10.17, \mathrm{n}: 8)$ and human wt $\alpha$-syn and GFP-DNAJB6 groups $(\% 61.99 \pm 5.951, \mathrm{n}: 8)$ (Figure 3A - E).

\section{Overexpression of DNAJB6 attenuate motor impairments}

To evaluate the gradual locomotor impairment caused by $\alpha$-syn overexpression and the potential protective effect of DNAJB6 expression, we performed stepping and cylinder tests on the animals before injection and at 4,8 and 12 weeks post-injection. The group expressing $\alpha$-syn and GFP in the SNpc showed gradually deterioration in stepping tests from 4 to 12 weeks. In comparison, the overexpression of DNAJB6 rescued to the motor behavior impairments of rats in stepping test. Figure 4A $(\mathrm{F}(3,42): 12.96, \mathrm{p}<0.0001)$ and Figure 4B $(\mathrm{F}(3,42): 6.658, \mathrm{p}: 0,0009)$. However, using cylinder test, we were not able to find any significant difference between groups. Figure 4C (F(3,42) :0.5468, p :0,6530)

Overexpression of DNAJB6 causes decrease in phosphorylated $\alpha$-syn (at serine129) in SN 
$\alpha$-syn toxicity is tightly linked to its ability to form aggregates and it has been shown that aggregated $\alpha$-syn proportionally more phosphorylated at S129 (29). Therefore, we stained the brain sections containing $S N p c$ with anti-TH and anti-pS129-syn antibodies (Figure 5A-H). We observed that the group that expressed $\alpha$-syn and GFP in $S N p c$ showed more p129-syn staining (Figure 5A-D) in the TH positive cells, compare to the group that expressed $\alpha$-syn and GFP-DNAJB6 (Figure 5E-H). This suggests that the overexpression of DNAJB6 may suppress $\alpha$-syn aggregation in this animal model.

\section{Discussion}

Here we demonstrate for the first time that expression of a DNAJ protein can impair disease pathology in an animal model of PD. increased aggregation of $\alpha$-syn is linked to PD pathology (30). It is therefore pivotal to find ways to decrease or prevent the $\alpha$-syn aggregation. HSP70 chaperones and the DNAJ co-chaperones are proteins that have important roles in protein folding as well as re-folding or clearance of misfolded proteins $(31,32)$. There is substantial evidence that HSP70's are involved in preventing aggregated $\alpha$-syn from accumulating. HSP70 may bind different $\alpha$-syn species, including monomer, pre-fibrillar and fibrillar forms, and disaggregates in vitro $(9,13-15,33,34)$. In vivo, overexpression of HSP70 reduced the amount of $\alpha$-syn aggregates, in mice overexpressing $\alpha$-syn (35). In addition, our lab has shown previously that DNAJB6 is particular important in this process and that DNAJB6 works HSP70 dependently $(19,20,36)$.

We have previously shown that KO of DNAJB6 increases $\alpha$-syn aggregation, which could be rescued by re-introduction of DNAJB6b into the cells, while we did not observe effect $\alpha$-syn aggregation by KO of the DNAJB6a isoform only (19). DNAJB6 is mainly localized in the cytosol while DNAJB6a is mainly found in the nucleus (37), which could explain why only DNAJB6b is a suppressor of $\alpha$-syn aggregation. Therefore, we proceeded these studies with a focus on exploring the role of DNAJB6b, rather than DNAJB6a, in cell culture and in vivo. In 
this study, we investigated the protective role of co-chaperone DNAJB6 against $\alpha$-syn aggregation and toxicity in a cell culture model as well rodent model, both based on $\alpha$-syn overexpression. $\alpha$-syn overexpression model has a relevant parallel for clinical cases of PD as well, as both duplication and triplication of the SNCA gene leads to a very high risk of contracting PD (38). We found that overexpression of DNAJB6b strongly suppressed aggregation of $\alpha$-syn the cellular model of PD (Figure 1). This finding supports our previous results in cellular models. Moreover, it is also in line with the results of other research labs, which suggests a general role of DNAJB6 in suppressing amyloid protein aggregation, as overexpression DNAJB6 protects from the formation of amyloid protein aggregates such as polyQ $(21,23,24)$ and AB42 (22) which are seen in Huntington's and Alzheimer's Disease, respectively. Indeed, we also found that $\mathrm{KO}$ of DNAJB6 in stably polyQ74exon1huntingtin (polyQ74) expressing HEK293 cells increased to polyQ74 aggregations and cell death (36). The effects of DNAJB6 on amyloid protein aggregation have been investigated mostly in cellular models. Therefore, our knowledge regarding effects of DNAJB6 on amyloid protein aggregation in terms of in vivo studies, are still limited. However, one study has shown that DNAJB6 delays disease progression in a mouse model of HD (24).

DNAJB6 is highly expressed in nervous tissue and interestingly the amount of DNAJB6 is substantially increased in LB's of PD patients (18). We do not yet know why DNAJB6 is found at higher levels in PD and this still remains to be explored. Our study is the first that show a protective role of DNAJ6 in an animal model of PD. We performed AAV mediated $\alpha$-syn overexpression to investigated protective effects of DNAJB6 in vivo. This model was originally designed by Kirik et al (39) and mimics some of neuropathological and behavioral impairments of PD. In this work, we have revealed for the first time that overexpression of DNAJB6 protects against cell death of the dopaminergic neurons in the $S N p c$ induced by $\alpha-$ syn overexpression (Figure 2 and 3). We did not observe a difference in the loss of striatal TH 
staining between the experimental groups, but this could be because of compensatory mechanisms in the surviving $\mathrm{TH}+$ neurons of the rats. However, as a consequence of the dopaminergic cell death, the rats showed impaired locomotor performance in stepping test which was progressive over a 12 week time course in the $\alpha$-syn + GFP expressing rats, but not in the $\alpha$-syn + GFP-DNAJB6 expressing rats (Figure 4). We did not see any behavioral differences in the cylinder test (Figure 4), but it is possible that in order to observe behavioral impairments in the cylinder test, then this requires a higher degree of dopaminergic cell death, than induced in this study. In line with this Thakur et al (40) has shown the functional deficits in the cylinder test was not caused by neither $\alpha$-syn overexpression nor injection of $\alpha$-syn PFF's into rats, but only in the case of a combination of the two, which also resulted in a substantially higher dopaminergic cell death

We found that the amount of total $\alpha$-syn was less in the $\alpha$-syn + GFP-DNAJB6 group of rats compared to the $\alpha$-syn + GFP group (Figure 3B and F). Our prior data suggest that DNAJB6 may suppress $\alpha$-syn aggregation through targeting it for proteasomal degradation, which could explain these results (20). HSP70 family of proteins, play a role in both main protein degradation pathways, ubiquitin-proteasome system and (UPS) and autophagy-lysosome pathway (ALP) (41). Recently, it has proposed that while UPS is effective to degrading smaller $\alpha$-syn species in young and healthy organisms, ALP is considered to be effective against larger $\alpha$-syn species in aging $(42,43)$. This theory, and the observations made by Kampinga and colleagues that DNAJB6 interferes with protein aggregation in an early phase (24), fits in line with that the mechanism by which DNAJB6 suppress $\alpha$-syn aggregation could be by targeting the smaller aggregates and delivering these to the HSP70 chaperones leading to refolding or targeting for proteosomal degradation.

In this study we observed that $\alpha$-syn was found phosphorylated at serine 129 in $S N p c$ of rats, to a larger extend in the rats that expressed $\alpha$-syn and GFP than the rats that expressed $\alpha$-syn 
and GFP-DNAJB6 (Figure 5). As aggregated $\alpha$-syn is in general more phosphorylated at Ser129 than monomeric $\alpha$-syn, this suggests, that DNAJB6b is a strong suppressor of $\alpha$-syn aggregation.

\section{Conclusion}

We have demonstrated that DNAJB6 is a strong suppressor of $\alpha$-syn aggregation in cell culture and in vivo and well as it protects against $\alpha$-syn induced pathology in a rodent model of PD. In future, compounds that specifically target the upregulation of DNAJB6 levels, or activity of the protein, may be good future drug candidates for treatment of early stage PD.

\section{List of Abbreviations}

AAV: Adeno associated virus, AB42: Amyloid beta-42, $\alpha$-syn: alpha synuclein, ALS: Autophagy-lysosome pathway, AP: Anteroposterior, CFP: Cyan fluorescence protein, DMEM: Dulbecco's modified eagle's medium with GlutaMAX-I, DV: Dorsoventral, EDTA: Ethylenediaminetetraacetic acid, FBS: Fetal Bovine Serum, FRET: Förster resonance energy transfer, GFP: Green fluorescence protein, HD: Hungtington disease, HSP70: Heat shock protein 70, IHC: Immunohistochemistry, LB: Lewy body, ML: Mediolateral, PBS: Phosphate Buffered Saline, PBS-T: Phosphate Buffered Saline with Tween 80, PD: Parkinson disease, PPF: Preformed fibril, polyQ74: polyQ74exon1huntingtin, P/S: penicillin/streptomycin, PVDF: Polyvinylidene fluorid, RT: Room temperature, SDS PAGE: Sodium dodecyl sulphate-polyacrylamide gel electrophoresis, SN: Substansia nigra, SNpc: Substansia nigra pars compacta, TBS: Tris buffered saline, TBS-T: Tris buffered saline with Triton X-100, TH: Tyrosine hydroxylase, UPS: Ubiquitin-proteasome system, YFP: Yellow fluorescence protein

\section{Declarations}

\section{Ethical Approval}

The studies were performed in accordance with the European Union Directive (2010/63/EU) and approved by the local ethical committee for the use of laboratory animals and the 
Swedish Department of Agriculture (Jordbruksverket (Skåne, Sweden), permit number 890118).

\section{Consent for publication}

Not applicable

Availability of data and materials

All data are used in this study are included in this article.

\section{Competing interests}

The authors declare no competing interests

\section{Funding}

The authors acknowledge that the financial support from the EU Joint programmeNeurodegenerative Disease, the Royal Physiographic Society of Lund (Project Number : 40411) and Tore Nilsson Stiftelse (Project Number: 2019-00727). Sertan Arkan was supported by Stiftelsen Olle Engkvist Byggmästare.

\section{Author Contributions}

Sertan Arkan contributed experimental design, execution experiments, data collection, data analysis and manuscript writing. Mårten Ljungberg execution experiments, contributed data collection and manuscript writing. Deniz Kirik contributed experimental design, data analysis and manuscript writing. Christian Hansen contributed experimental design, data collection, data analysis and manuscript writing. All authors read and approved the final manuscript.

\section{Acknowledgement}

The authors wish to thank Anneli Josefsson, Ulla Samuelsson and Björn Anzelius for their excellent technical support

\section{References}

1. Goedert M, Spillantini MG, Del Tredici K, Braak H. 100 years of Lewy pathology. Nat Rev Neurol. 2013;9(1):13-24. 
2. Hansen C, Li JY. Beyond alpha-synuclein transfer: pathology propagation in Parkinson's disease. Trends Mol Med. 2012;18(5):248-55.

3. Vekrellis K, Xilouri M, Emmanouilidou E, Rideout HJ, Stefanis L. Pathological roles of alpha-synuclein in neurological disorders. Lancet Neurol. 2011;10(11):1015-25.

4. Waxman EA, Giasson BI. Molecular mechanisms of alpha-synuclein neurodegeneration. Biochim Biophys Acta. 2009;1792(7):616-24.

5. Spillantini MG, Schmidt ML, Lee VM, Trojanowski JQ, Jakes R, Goedert M. Alphasynuclein in Lewy bodies. Nature. 1997;388(6645):839-40.

6. Bonini NM, Giasson BI. Snaring the function of alpha-synuclein. Cell. 2005;123(3):359-61.

7. Burre J, Sharma M, Tsetsenis T, Buchman V, Etherton MR, Sudhof TC. Alpha-synuclein promotes SNARE-complex assembly in vivo and in vitro. Science. 2010;329(5999):1663-7.

8. Logan T, Bendor J, Toupin C, Thorn K, Edwards RH. alpha-Synuclein promotes dilation of the exocytotic fusion pore. Nat Neurosci. 2017;20(5):681-9.

9. Auluck PK, Chan HY, Trojanowski JQ, Lee VM, Bonini NM. Chaperone suppression of alpha-synuclein toxicity in a Drosophila model for Parkinson's disease. Science.

2002;295(5556):865-8.

10. Balch WE, Morimoto RI, Dillin A, Kelly JW. Adapting proteostasis for disease intervention. Science. 2008;319(5865):916-9.

11. Cuervo AM, Stefanis L, Fredenburg R, Lansbury PT, Sulzer D. Impaired degradation of mutant alpha-synuclein by chaperone-mediated autophagy. Science. 2004;305(5688):12925.

12. Hartl FU, Bracher A, Hayer-Hartl M. Molecular chaperones in protein folding and proteostasis. Nature. 2011;475(7356):324-32.

13. Aprile FA, Sormanni P, Vendruscolo M. A Rational Design Strategy for the Selective Activity Enhancement of a Molecular Chaperone toward a Target Substrate. Biochemistry. 2015;54(32):5103-12.

14. Gao X, Carroni M, Nussbaum-Krammer C, Mogk A, Nillegoda NB, Szlachcic A, et al. Human Hsp70 Disaggregase Reverses Parkinson's-Linked alpha-Synuclein Amyloid Fibrils. Mol Cell. 2015;59(5):781-93.

15. McLean PJ, Klucken J, Shin Y, Hyman BT. Geldanamycin induces Hsp70 and prevents alpha-synuclein aggregation and toxicity in vitro. Biochem Biophys Res Commun. 2004;321(3):665-9.

16. De Mattos EP, Wentink A, Nussbaum-Krammer C, Hansen C, Bergink S, Melki R, et al. Protein Quality Control Pathways at the Crossroad of Synucleinopathies. J Parkinsons Dis. 2020;10(2):369-82.

17. Qiu XB, Shao YM, Miao S, Wang L. The diversity of the DnaJ/Hsp40 family, the crucial partners for Hsp70 chaperones. Cell Mol Life Sci. 2006;63(22):2560-70.

18. Durrenberger PF, Filiou MD, Moran LB, Michael GJ, Novoselov S, Cheetham ME, et al. DnaJB6 is present in the core of Lewy bodies and is highly up-regulated in parkinsonian astrocytes. J Neurosci Res. 2009;87(1):238-45.

19. Aprile FA, Kallstig E, Limorenko G, Vendruscolo M, Ron D, Hansen C. The molecular chaperones DNAJB6 and Hsp70 cooperate to suppress alpha-synuclein aggregation. Sci Rep. 2017;7(1):9039.

20. Deshayes N, Arkan S, Hansen C. The Molecular Chaperone DNAJB6, but Not DNAJB1, Suppresses the Seeded Aggregation of Alpha-Synuclein in Cells. Int J Mol Sci. 2019;20(18). 
21. Gillis J, Schipper-Krom S, Juenemann K, Gruber A, Coolen S, van den Nieuwendijk R, et al. The DNAJB6 and DNAJB8 protein chaperones prevent intracellular aggregation of polyglutamine peptides. J Biol Chem. 2013;288(24):17225-37.

22. Mansson C, Arosio P, Hussein R, Kampinga HH, Hashem RM, Boelens WC, et al. Interaction of the molecular chaperone DNAJB6 with growing amyloid-beta 42 (Abeta42) aggregates leads to sub-stoichiometric inhibition of amyloid formation. J Biol Chem. 2014;289(45):31066-76.

23. Mansson C, Kakkar V, Monsellier E, Sourigues Y, Harmark J, Kampinga HH, et al. DNAJB6 is a peptide-binding chaperone which can suppress amyloid fibrillation of polyglutamine peptides at substoichiometric molar ratios. Cell Stress Chaperones. 2014;19(2):227-39.

24. Kakkar V, Mansson C, de Mattos EP, Bergink S, van der Zwaag M, van Waarde M, et al. The S/T-Rich Motif in the DNAJB6 Chaperone Delays Polyglutamine Aggregation and the Onset of Disease in a Mouse Model. Mol Cell. 2016;62(2):272-83.

25. Holmes BB, Furman JL, Mahan TE, Yamasaki TR, Mirbaha H, Eades WC, et al. Proteopathic tau seeding predicts tauopathy in vivo. Proc Natl Acad Sci U S A. 2014;111(41):E4376-85.

26. Landeck N, Hall H, Ardah MT, Majbour NK, El-Agnaf OM, Halliday G, et al. A novel multiplex assay for simultaneous quantification of total and S129 phosphorylated human alpha-synuclein. Mol Neurodegener. 2016;11(1):61.

27. Ulusoy A, Bjorklund T, Buck K, Kirik D. Dysregulated dopamine storage increases the vulnerability to alpha-synuclein in nigral neurons. Neurobiol Dis. 2012;47(3):367-77.

28. Kirik D, Rosenblad C, Bjorklund A, Mandel RJ. Long-term rAAV-mediated gene transfer of GDNF in the rat Parkinson's model: intrastriatal but not intranigral transduction promotes functional regeneration in the lesioned nigrostriatal system. J Neurosci.

2000;20(12):4686-700.

29. Fujiwara H, Hasegawa M, Dohmae N, Kawashima A, Masliah E, Goldberg MS, et al. alpha-Synuclein is phosphorylated in synucleinopathy lesions. Nat Cell Biol. 2002;4(2):160-4. 30. Venda LL, Cragg SJ, Buchman VL, Wade-Martins R. alpha-Synuclein and dopamine at the crossroads of Parkinson's disease. Trends Neurosci. 2010;33(12):559-68.

31. Jones DR, Moussaud S, McLean P. Targeting heat shock proteins to modulate alphasynuclein toxicity. Ther Adv Neurol Disord. 2014;7(1):33-51.

32. Hartl FU. Molecular chaperones in cellular protein folding. Nature. 1996;381(6583):571-9.

33. Nillegoda NB, Bukau B. Metazoan Hsp70-based protein disaggregases: emergence and mechanisms. Front Mol Biosci. 2015;2:57.

34. Rampelt H, Kirstein-Miles J, Nillegoda NB, Chi K, Scholz SR, Morimoto RI, et al. Metazoan Hsp70 machines use Hsp110 to power protein disaggregation. EMBO J. 2012;31(21):4221-35.

35. Klucken J, Shin Y, Masliah E, Hyman BT, McLean PJ. Hsp70 Reduces alpha-Synuclein Aggregation and Toxicity. J Biol Chem. 2004;279(24):25497-502.

36. Rodriguez-Gonzalez C, Lin S, Arkan S, Hansen C. Co-chaperones DNAJA1 and DNAJB6 are critical for regulation of polyglutamine aggregation. Sci Rep. 2020;10(1):8130.

37. Meng E, Shevde LA, Samant RS. Emerging roles and underlying molecular mechanisms of DNAJB6 in cancer. Oncotarget. 2016;7(33):53984-96. 
38. Ross OA, Braithwaite AT, Skipper LM, Kachergus J, Hulihan MM, Middleton FA, et al. Genomic investigation of alpha-synuclein multiplication and parkinsonism. Ann Neurol. 2008;63(6):743-50.

39. Kirik D, Rosenblad C, Burger C, Lundberg C, Johansen TE, Muzyczka N, et al. Parkinson-like neurodegeneration induced by targeted overexpression of alpha-synuclein in the nigrostriatal system. J Neurosci. 2002;22(7):2780-91.

40. Thakur P, Breger LS, Lundblad M, Wan OW, Mattsson B, Luk KC, et al. Modeling Parkinson's disease pathology by combination of fibril seeds and alpha-synuclein overexpression in the rat brain. Proc Natl Acad Sci U S A. 2017;114(39):E8284-E93.

41. Ciechanover A, Kwon YT. Protein Quality Control by Molecular Chaperones in Neurodegeneration. Front Neurosci. 2017;11:185.

42. Ebrahimi-Fakhari D, Cantuti-Castelvetri I, Fan Z, Rockenstein E, Masliah E, Hyman BT, et al. Distinct roles in vivo for the ubiquitin-proteasome system and the autophagylysosomal pathway in the degradation of alpha-synuclein. J Neurosci. 2011;31(41):14508-20. 43. Emmanouilidou E, Stefanis L, Vekrellis K. Cell-produced alpha-synuclein oligomers are targeted to, and impair, the 26S proteasome. Neurobiol Aging. 2010;31(6):953-68.

\section{Figure Legends}

Figure 1: Overexpression of DNAJB6 leads to suppression of $\alpha$-syn in $\alpha$-syn-CFP, $\alpha$ syn-YFP HEK293 cell line. (A) Percentage of cells displaying FRET signal, expressing DNAJB6-mCherry or mCherry (control), 72 hours subsequent to plasmid transfection and 48 hours subsequent to inducing $\alpha$-syn aggregation with 10nM $\alpha$-syn PFF's (n=3). (B) Western blot displaying expression of DNAJB6b in lysates from $\alpha$-syn FRET HEK293 cells transfected with DNAJB6b or mCherry transfected control cells. Membrane was probed with anti-DNAJB6 and anti-actin antibodies. All data are presented as mean $\pm \mathrm{SEM} . \quad * * * \mathrm{P}<$ 0.001. Data was analyzed by unpaired t-test.

Figure 2: DNAJB6 expression protects against $\alpha$-syn induced loss of dopaminergic neurons in the $\boldsymbol{S N p c}$. Representative pictures of $S N p c$ stainings with anti-TH (purple) and anti- $\alpha$-syn (red) combined with GFP fluorescence (green) in $40 \mu \mathrm{M}$ brain tissue sections from rats injected with either AAV6/ human wt $\alpha$-syn and AAV6/GFP vectors (A-D) or AAV6/ human wt $\alpha$-syn and AAV6/GFP-DNAJB6 vectors (E-H). Representative images of $S N p c$ from rats injected with AAV6/ human wt $\alpha$-syn and AAV6/GFP stained with anti-TH antibody, depicting the contralateral (I) and the ipsilateral (J) $S N p c$ of the injected rats, with 
the ipsilateral side displaying less $\mathrm{TH}+$ neurons $(\% 33.62 \pm 7.404, \mathrm{n}=8)(\mathrm{J})$. Representative images of $S N p c$ from rats injected with AAV6/ $\alpha$-syn and AAV6/GFP-DNAJB6 stained with with anti-TH antibody, depicting the contralateral (K) and the ipsilateral (L) SNpc of the injected rats, with the ipsilateral side displaying less $\mathrm{TH}+$ neurons $(\% 61.34 \pm 8.162, \mathrm{n}=8)$. (M) Quantification of $\mathrm{TH}+$ cell number in the $S N p c$, which are expressed as a percentage of the contralateral side $(\mathrm{P}<0.05)$ analyzed by unpaired $\mathrm{t}$-test $(\mathrm{df}=14, \mathrm{t}=2.515, \mathrm{p}=0.0247)$. All data are presented as mean \pm SEM. Scale bars, $100 \mu \mathrm{m}$.

\section{Figure 3: Representative images of TH immunohistochemistry staining in AAV6 vira} injected rats. Representative images of Striatum (A) and $S N(\mathrm{C})$ of AAV6/ human wt $\alpha$-syn and AAV6/GFP injected rats. Representative images of striatum (B) and $S N$ (D) of AAV6 human wt $\alpha$-syn and GFP-DNAJB6 injected rats. (E) Quantification of TH+ fiber density in the striatum, expressed as a percentage of the contralateral side. $\mathrm{P}>0.05$ analyzed by unpaired$\mathrm{t}$-test (n:8). All data are presented as mean \pm SEM. (Scale bar, $1 \mathrm{~mm}$ )

Figure 4: DNAJB6 expression protects against behavioral deficits induced by human wt $\alpha$-syn, as seen in stepping test analysis of vira injected rats . Locomotor performance was assessed using the stepping test (A and B) and cylinder test (C) at 4,8 and 12 week time points. (A) display left forehand stepping relative percentage to total steps. (B) display left forehand steps relative to percentage of right forehand steps (C) In cylinder test, left paw touches relative to percentage of total touches were analyzed. All Data were analyzed by repeated measure two-way ANOVA, followed by post hoc Bonferroni test. All data are presented as mean \pm SEM. $* \mathrm{P}<0.05, * * * \mathrm{P}<0.001$

Figure 5 : S129 phosphorylation of $\alpha$-syn is higher in human wt $\alpha$-syn + GFP injected rats compared to human wt $\boldsymbol{\alpha}$-syn + GFP-DNAJB6 injected rats. Representative images of $S N p c$ from rats injected with AAV6/ human wt $\alpha$-syn and AAV6/GFP vira (A-D) or AAV6/ 
human wt $\alpha$-syn and AAV6/GFP-DNAJB6 vectors (E-H), depicting staining of pS129 $\alpha$-syn (red), TH (purple) and GFP fluorescence (green). (Scale bar $50 \mu \mathrm{m}$ ) 
A

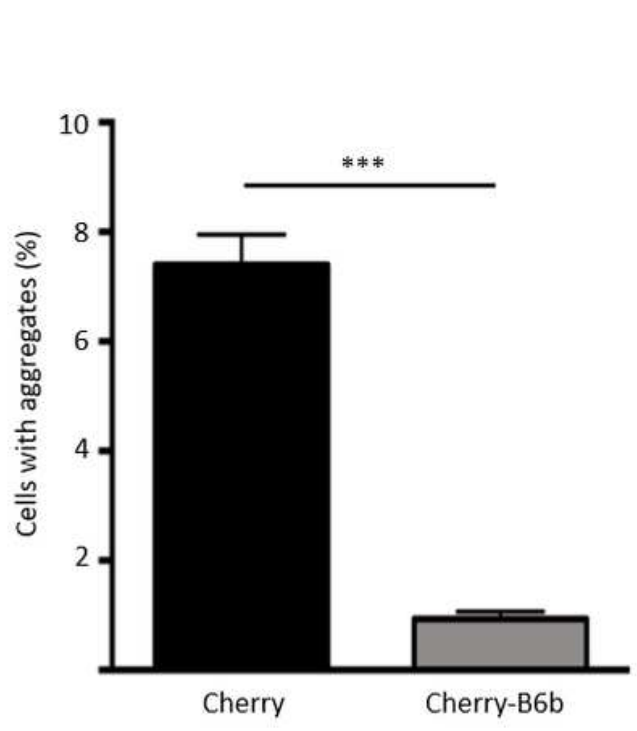

B

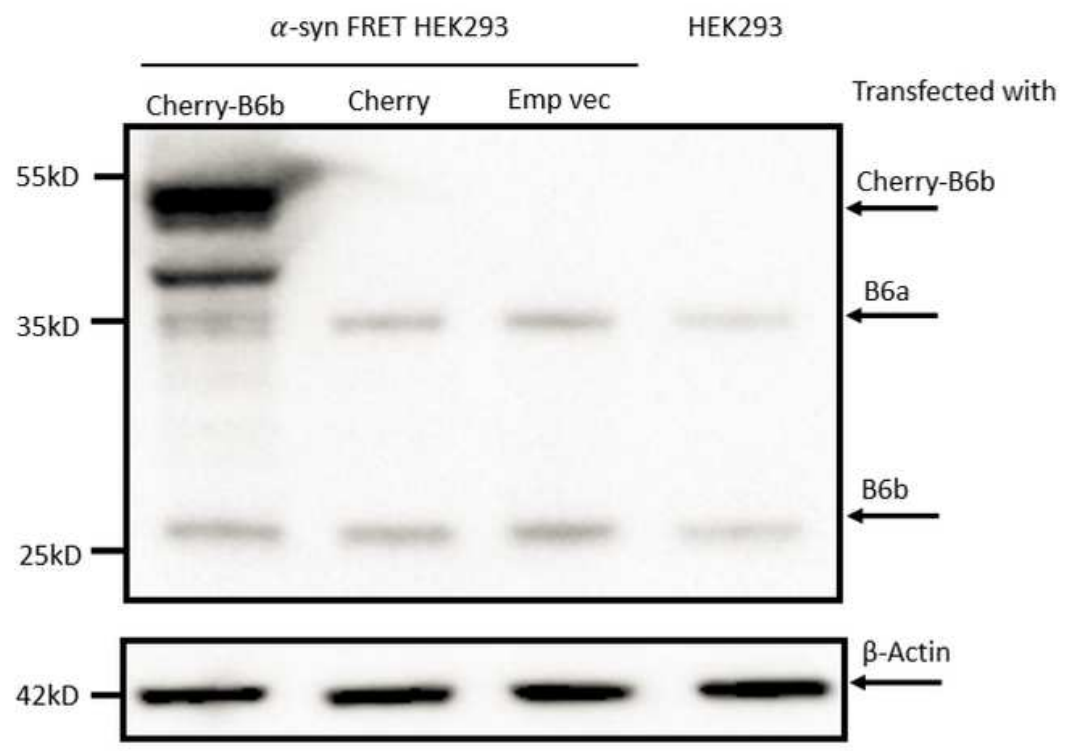

Figure 1

Overexpression of DNAJB6 leads to suppression of a-syn in a-syn-CFP, a syn-YFP HEK293 cell line. (A) Percentage of cells displaying FRET signal, expressing DNAJB6-mCherry or mCherry (control), 72 hours subsequent to plasmid transfection and 48 hours subsequent to inducing a-syn aggregation with $10 \mathrm{nM}$ a-syn PFF's ( $n=3)$. (B) Western blot displaying expression of DNAJB6b in lysates from a-syn FRET HEK293 cells transfected with DNAJB6b or mCherry transfected control cells. Membrane was probed with anti-DNAJB6 and anti-actin antibodies. All data are presented as mean \pm SEM. ${ }^{\star \star \star} P \mathrm{P}<0.001$. Data was analyzed by unpaired t-test. 

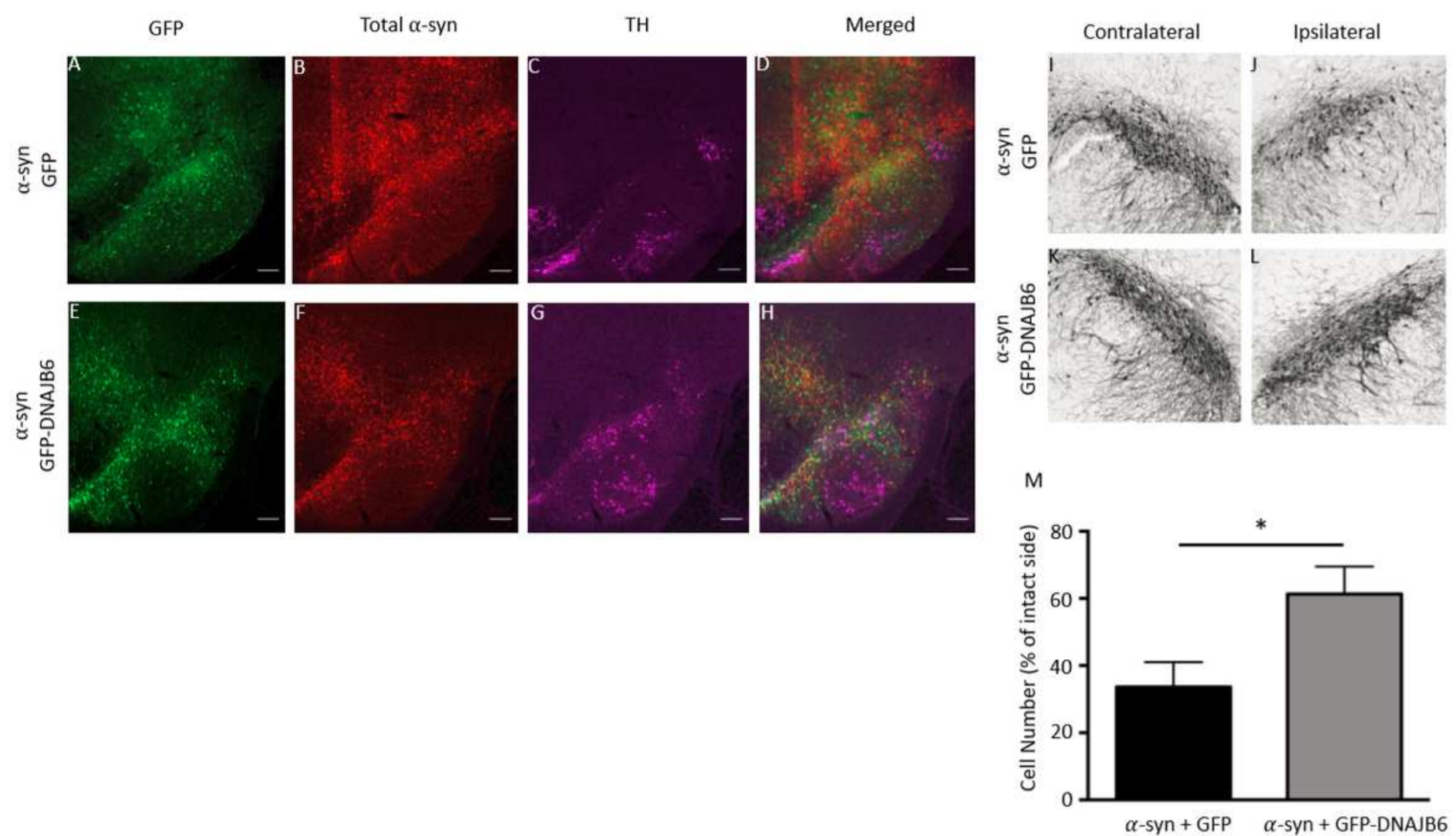

Figure 2

DNAJB6 expression protects against a-syn induced loss of dopaminergic neurons in the SNpc.

Representative pictures of SNpc stainings with anti-TH (purple) and anti-a-syn (red) combined with GFP fluorescence (green) in $40 \mu \mathrm{M}$ brain tissue sections from rats injected with either AAV6/ human wt a-syn and AAV6/GFP vectors (A-D) or AAV6/ human wt a-syn and AAV6/GFP-DNAJB6 vectors (E-H).

Representative images of SNpc from rats injected with AAV6/ human wt a-syn and AAV6/GFP stained with anti-TH antibody, depicting the contralateral $(I)$ and the ipsilateral $(J)$ SNpc of the injected rats, with the ipsilateral side displaying less $\mathrm{TH}+$ neurons $(\% 33.62 \pm 7.404, \mathrm{n}=8)(\mathrm{J})$. Representative images of SNpc from rats injected with AAV6/a-syn and AAV6/GFP-DNAJB6 stained with with anti-TH antibody, depicting the contralateral $(K)$ and the ipsilateral $(L) S N p c$ of the injected rats, with the ipsilateral side displaying less $\mathrm{TH}+$ neurons $(\% 61.34 \pm 8.162, \mathrm{n}=8)$. (M) Quantification of $\mathrm{TH}+$ cell number in the SNpc, which are expressed as a percentage of the contralateral side $(P<0.05)$ analyzed by unpaired $t$-test $(d f=14, t=$ $2.515, p=0.0247)$. All data are presented as mean \pm SEM. Scale bars, $100 \mu \mathrm{m}$. 
A

B

$\alpha$-syn + GFP

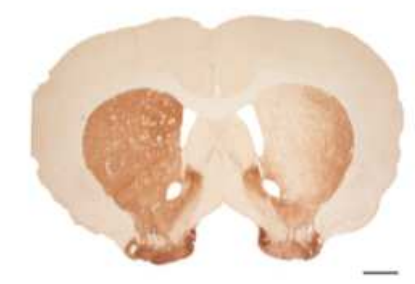

C

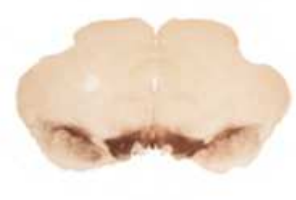

$\alpha$-syn + GFP-DNAJB6

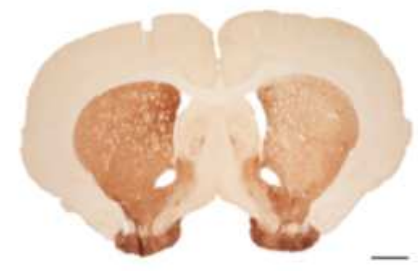

D

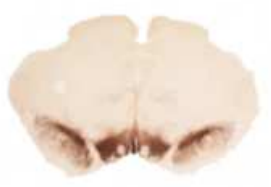

$\mathrm{E}$

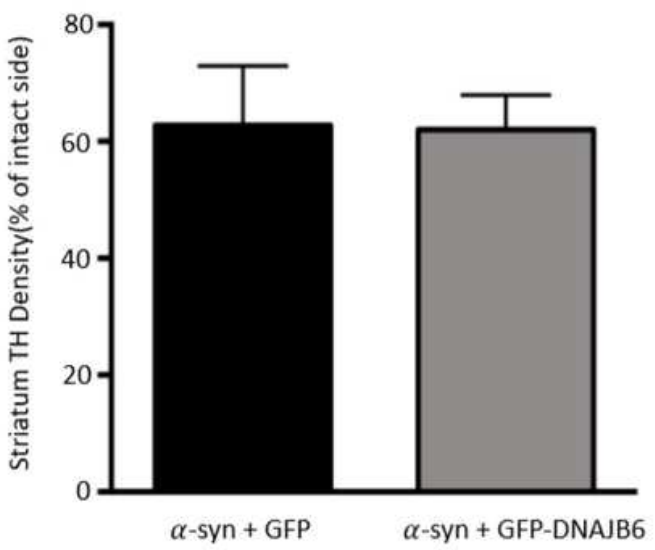

\section{Figure 3}

Representative images of TH immunohistochemistry staining in AAV6 vira injected rats. Representative images of Striatum (A) and SN (C) of AAV6/ human wt a-syn and AAV6/GFP injected rats. Representative images of striatum (B) and SN (D) of AAV6 human wt a-syn and GFP-DNAJB6 injected rats. (E) Quantification of $\mathrm{TH}+$ fiber density in the striatum, expressed as a percentage of the contralateral side. $P>0.05$ analyzed by unpaired t-test $(n: 8)$. All data are presented as mean $\pm S E M$. (Scale bar, $1 \mathrm{~mm}$ )

A

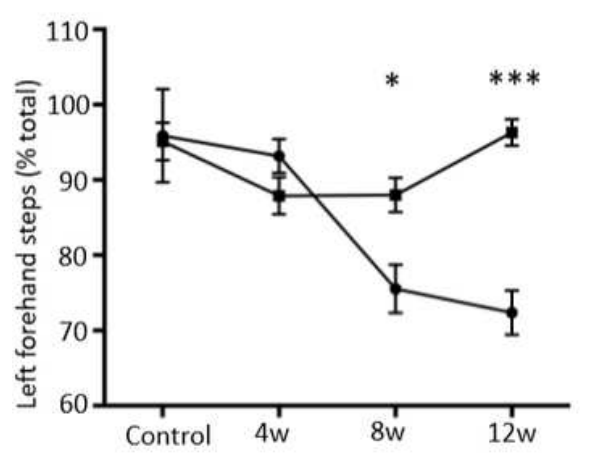

B

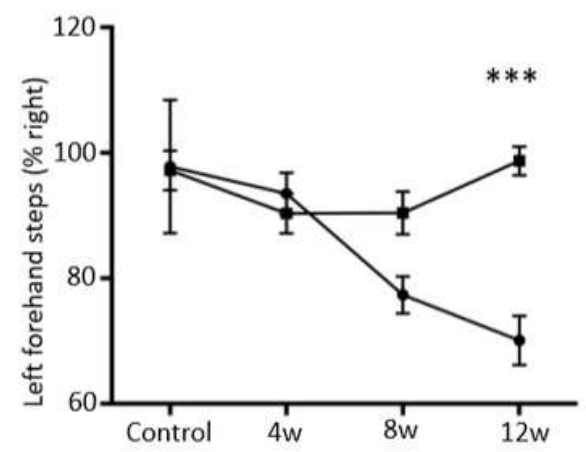

C

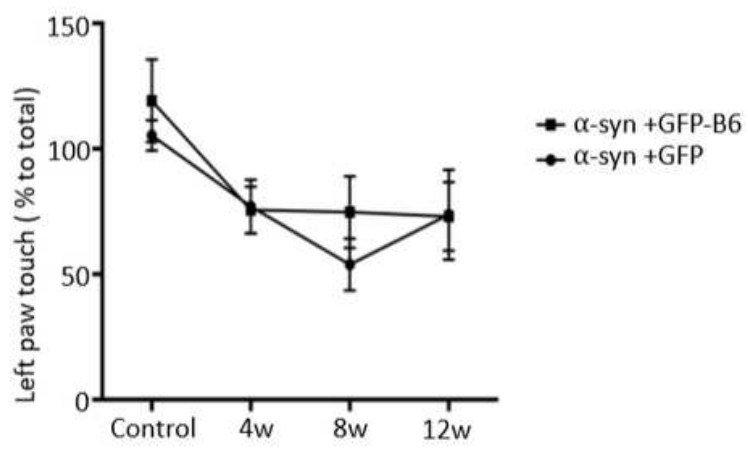

Figure 4

DNAJB6 expression protects against behavioral deficits induced by human wt a-syn, as seen in stepping test analysis of vira injected rats . Locomotor performance was assessed using the stepping test (A and $B$ ) and cylinder test (C) at 4, 8 and 12 week time points. (A) display left forehand stepping relative percentage to total steps. (B) display left forehand steps relative to percentage of right forehand steps (C) 
In cylinder test, left paw touches relative to percentage of total touches were analyzed. All Data were analyzed by repeated measure two-way ANOVA, followed by post hoc Bonferroni test. All data are presented as mean \pm SEM. ${ }^{*} \mathrm{P}<0.05,{ }^{\star \star *} \mathrm{P}<0.001$

GFP

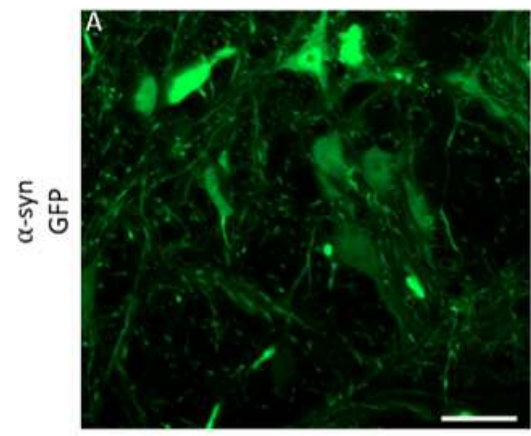

(3)

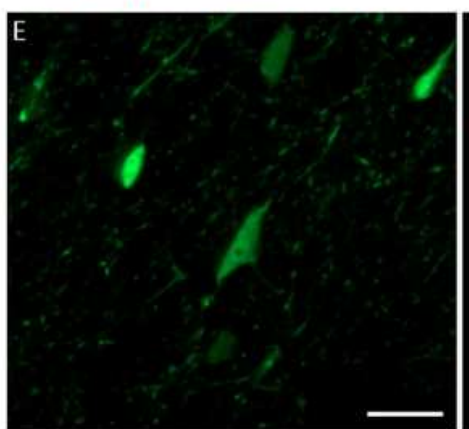

pSer129- $\alpha$ syn
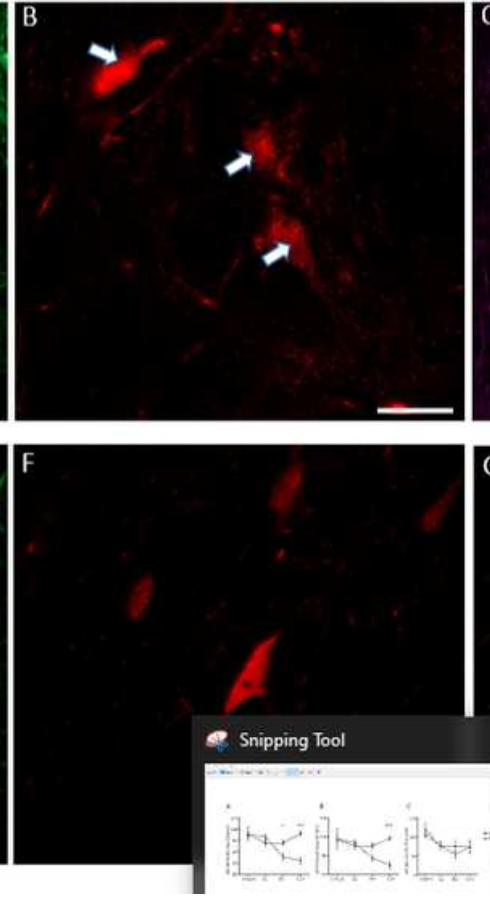

$\mathrm{TH}$

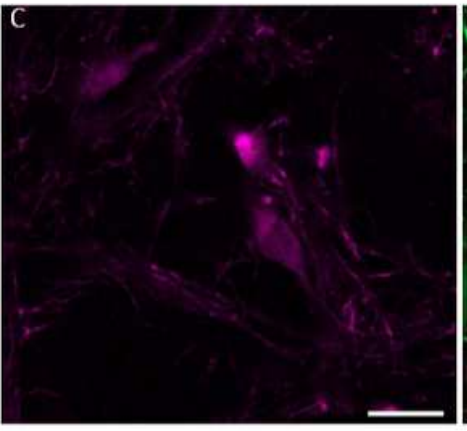

G

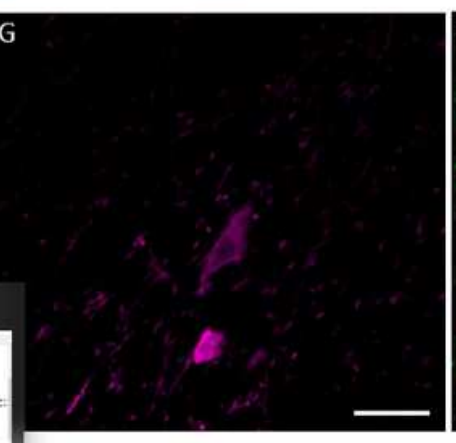

Merged
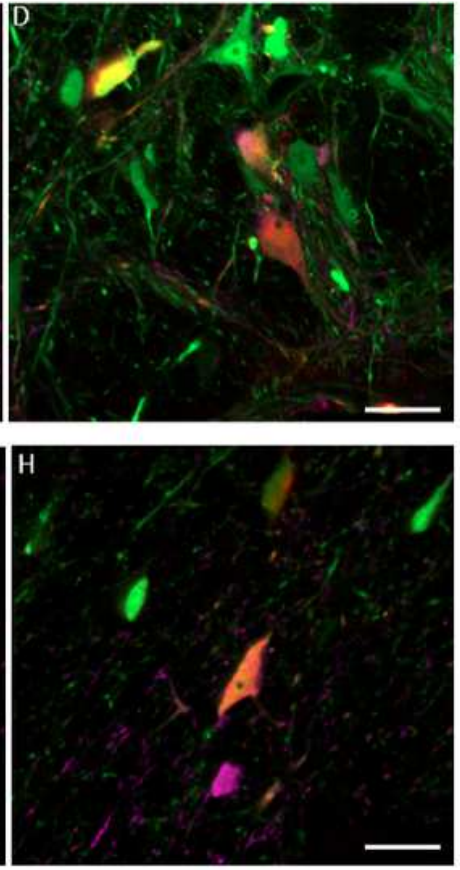

\section{Figure 5}

S129 phosphorylation of a-syn is higher in human wt a-syn + GFP injected rats compared to human wt asyn + GFP-DNAJB6 injected rats. Representative images of SNpc from rats injected with AAV6/ human wt a-syn and AAV6/GFP vira (A-D) or AAV6/ human wt a-syn and AAV6/GFP-DNAJB6 vectors (E-H), depicting staining of pS129 a-syn (red), TH (purple) and GFP fluorescence (green). (Scale bar $50 \mu \mathrm{m}$ ) 